\section{Ejaculatio praecox operativ begegnen}

Der vorzeitige Samenerguss ist einer der häufigsten Sexualstörungen bei Männern. Mit einer selektiven Resektion der dorsalen Penisnerven gelang es chinesischen Urologen nun, die intravaginale Latenzzeit zu verlängern und die Ejakulationskontrolle zu erhöhen.

n einer Studie mit 101 Männern im Alter zwischen 18 und 35 Jahren, die an Ejaculatio praecox litten, haben chinesische Urologen ein operatives Verfahren im Zuge einer randomisierten Studie erprobt. Alle Probanden unterzogen sich einer elektiven Zirkumzision, bei 41 von ihnen wurden im Anschluss daran die dorsalen Nerven des Penis selektiv reseziert.

Die intravaginale Ejakulationslatenzzeit verlängerte sich nach der Resektion von durchschnittlich 1,1 Minuten vor der Operation auf 3,8 Minuten nach dem Eingriff $(p<0,01)$. In der Kontrollgruppe, bei der es bei der einfachen Beschneidung geblieben war, gab es keine Veränderungen (1,2 vs. 1,5 Minuten; p > 0,05). Auch äußerten die Männer nach der Nervenentfernung, sie könnten den Erguss besser kontrollieren. Erektile Funktion und sexuelle Zufriedenheit blieben von der Resektion unbeeinflusst.

Bei dem Eingriff folgt auf die Zirkumzision und vor dem Vernähen des inneren und äußeren Präputialblatts eine Inzision von 10 bis 2 Uhr (Steinschnittlage) in die Fascia profunda des Penisschafts unterhalb der Corona glandis. Die distalen dorsalen Nervenäste werden präpariert, bei jedem zweiten Ast wird im Bereich des Sulcus coronarius glandis ein Segment von $0,5 \mathrm{~cm}$ Länge reseziert. Danach wird die Inzision verschlossen und die Operation mit dem Vernähen der Präputialblätter beendet. Die Idee hinter dem Eingriff ist es, durch die Resektion von Nervengewebe die Glans penis unempfindlicher zu machen. Dabei geht man von der Hypothese aus, dass die Hypersensitivität der Eichel zur vorzeitigen Ejakulation führt oder zumindest beiträgt.

Fazit: Mit einer selektiven Resektion der dorsalen Penisnerven lässt sich die Glans penis unempfindlicher machen und so bei Männern, die unter Ejaculatio praecox leiden, die intravaginale Latenzzeit verlängern

Dr. Robert Bublak

Zhang GX et al. Selective resection of dorsal nerves of penis for premature ejaculation. Int J Androl 2012; 35: 873-9

\section{Intermittierende Androgendeprivation: mehr Lust, weniger Flushs}

\section{Weitere Daten zur intermittierenden Androgenentzugstherapie beim Prostatakarzinom: In einer aktuellen Studie waren die Mortalitätsraten gegenüber einer kontinuierlich verabreichten antiandrogenen Therapie nicht erhöht. Dabei wurde nur ein Drittel der nebenwirkungsträchtigen LHRH-Agonisten benötigt.}

Stem teigen bei Prostatakarzinompatienten die PSA-Werte nach einer Strahlentherapie folgt in der Regel eine Androgendeprivation mittels GnRH-Agonisten oder -Blockern. Auch wenn den Männern die „echte“ Kastration erspart bleibt, müssen sie dennoch mit Nebenwirkungen wie Typ-2-Diabetes, koronarer Herzkrankheit oder Knochenbrüchen rechnen. Um solche toxischen Effekte zu reduzieren, wird in letzter Zeit verstärkt mit intermittierenden Therapiezyklen gearbeitet. Die Ergebnisse der bislang längsten und umfangreichsten klinischen Studie hierzu mit insgesamt 1.386 Patienten hat nun eine kanadische Forschungsgruppe vorgelegt.

Im direkten Vergleich der Gesamtüberlebensraten schnitt die Intervalltherapie nicht schlechter ab als die kontinuierliche Androgensuppression: Innerhalb von knapp sieben Jahren waren 268 von 690 Patienten mit Intervalltherapie und 256 von 696 Patienten mit kontinuierlicher Therapie verstorben. Das relative Mortalitätsrisiko der intermittierenden gegenüber der kontinuierlichen Therapie lag bei 1,03. Am Prostatakrebs selbst starben insgesamt „nur“ $14 \%$ der Teilnehmer. Der überwiegenden Mehrheit der Todesfälle lag also nach sieben Jahren eine andere Ursache zugrunde. Der Vergleich der beiden Gruppen zeigte dabei keinen signifikanten Unterschied. Die mit der Krankheit assoziierten Sterberaten lagen bei $18 \%$ beziehungsweise $15 \%$.

Überraschenderweise hielten sich die Nebenwirkungen der beiden therapeutischen Strategien die Waage. Auch die Gesamtscores zur Lebensqualität unterschieden sich kaum; nur in Einzelbereichen schienen die Teilnehmer der In- tervallgruppe zu profitieren: Sie hatten deutlich weniger Flush-Symptome und mehr Lust, sexuell aktiv zu sein ( $\mathrm{p}<0,001)$, litten weniger unter Blasenproblemen $(\mathrm{p}=0,006)$ und waren tendenziell auch weniger müde $(p=0,07)$. Dies hing jedoch möglicherweise mit dem Timing der Befragung zusammen.

Während die kontinuierlich behandelten Teilnehmer im Schnitt über 44 Monate Injektionen mit einem GnRH-Agonisten erhielten, lag die Gesamttherapiedauer in der Intervallgruppe bei gut 15 Monaten, insgesamt etwa drei Jahre blieben therapiefrei. Die Intervalle bestanden jeweils aus achtmonatigen Therapiezyklen. Die Dauer der dazwischenliegenden Injektionspausen richtete sich nach dem PSAWert (die Schwelle lag bei $10 \mathrm{ng} / \mathrm{ml}$ ).

Fazit: Die intermittierende Androgendeprivation war einer kontinuierlichen Therapie in allen untersuchten Überlebensparametern nicht unterlegen, bei gleichzeitig etwas geringeren Nebenwirkungen und deutlich niedrigeren Therapiekosten.

Dr. Elke Oberhofer

Crook JM et al. Intermittent Androgen Suppression for Rising PSA Level after Radiotherapy. N Engl J Med 2012; 367: 895-903 